\title{
Cross-Gramian-based dominant subspaces
}

\section{Peter Benner ${ }^{1,2}$ (1) $\cdot$ Christian Himpe ${ }^{1}$ (1)}

Received: 21 September 2018 / Accepted: 4 September 2019 /

Published online: 19 November 2019

(C) The Author(s) 2019

\begin{abstract}
A standard approach for model reduction of linear input-output systems is balanced truncation, which is based on the controllability and observability properties of the underlying system. The related dominant subspaces projection model reduction method similarly utilizes these system properties, yet instead of balancing, the associated subspaces are directly conjoined. In this work, we extend the dominant subspace approach by computation via the cross Gramian for linear systems, and describe an apriori error indicator for this method. Furthermore, efficient computation is discussed alongside numerical examples illustrating these findings.
\end{abstract}

Keywords Controllability · Observability - Cross Gramian · Model reduction · Dominant subspaces · HAPOD · DSPMR

Mathematics Subject Classification (2010) 93A15 · 93B11 · 93B20

\section{Introduction}

Input-output systems map an input function to an output function via a dynamical system. The input excites or perturbs the state of the dynamical system and the output is some transformation of the state. Typically, these input and output functions are low dimensional while the intermediate dynamical system is high(er) dimensional. In

Communicated by: Anthony Nouy

Christian Himpe

himpe@mpi-magdeburg.mpg.de

Peter Benner

benner@mpi-magdeburg.mpg.de

1 Computational Methods in Systems and Control Theory, Max Planck Institute for Dynamics of Complex Technical Systems, Sandtorstr. 1, 39106 Magdeburg, Germany

2 Faculty of Mathematics, Otto von Guericke University Magdeburg, Universitätsplatz 2, 39106 Magdeburg, Germany 
applications from natural sciences and engineering, the dimensionality of the dynamical system may render the numerical computation of outputs from inputs excessively expensive or at least demanding.

Model reduction addresses this computational challenge by algorithms that provide surrogate systems, which approximate the input-output mapping of the original system with a low(er) dimensional intermediate dynamical system. Practically, the trajectory of the dynamical system's state is constrained to a subspace of the original system's state-space, for example, by using truncated projections.

A standard approach for projection-based model reduction of input-output systems is balanced truncation [27], which transforms the state-space unitarily to a representation that is sorted (balanced) in terms of the input's effect on the state (controllability) as well as the state's effect on the output (observability) and discards (truncates) the least important states according to this measure.

Instead of balancing, this work investigates a dominant subspaces approach [32] that conjoins the most controllable and most observable subspaces into a projection. This unbalanced model reduction method may yield larger or less accurate reduced order systems, yet allows a computationally advantageous formulation while also preserving stability and providing an error quantification. The dominant subspace model reduction method has been investigated in [2, 23, 24, 32, 39], with [32] being the original source which is already referenced by the earlier work [24].

The approach proposed in this work combines the method from [32] with the cross Gramian (matrix) [13], which encodes controllability and observability information of an underlying input-output system. For this cross-Gramian-based dominant subspace method, an a-priori error indicator is developed, and the numerical issues arising in the wake of large-scale systems are addressed, specifically by utilizing the hierarchical approximate proper orthogonal decomposition (HAPOD) [18]. Compared to other cross Gramian and SVD model reduction techniques such as [22]; the proposed method does not need multiple decompositions, but a single HAPOD.

The considered class of input-output systems are generalized linear (time-invariant) systems $^{1}$, mapping input $u: \mathbb{R} \rightarrow \mathbb{R}^{M}$ via the state $x: \mathbb{R} \rightarrow \mathbb{R}^{N}$-a solution to an ordinary differential equation-to the output $y: \mathbb{R} \rightarrow \mathbb{R}^{Q}:$

$$
\begin{aligned}
E \dot{x}(t) & =A x(t)+B u(t), \\
y(t) & =C x(t),
\end{aligned}
$$

with a system matrix $A \in \mathbb{R}^{N \times N}$, an input matrix $B \in \mathbb{R}^{N \times M}$, an output matrix $C \in \mathbb{R}^{Q \times N}$, and a mass matrix $E \in \mathbb{R}^{N \times N}$. In the scope of this work, we assume $E$ to be non-singular as well as the matrix pencil $(A, E)$ to be asymptotically stable, meaning the eigenvalues of the associated generalized eigenproblem lie in the open left half-plane. This type of system arises, for example, in spatial discretizations of partial differential equations using the finite element method.

\footnotetext{
${ }^{1}$ Sometimes, the term descriptor system is used for this type of system, yet typically descriptor systems explicitly allow a singular mass matrix. Hence, we decided to use the term generalized linear system.
} 
In Section 2, the cross Gramian for generalized linear systems is introduced, followed by Section 3, briefly describing projection-based model reduction, and extending the dominant subspace projection method to the cross Gramian together with an error indicator. The proposed model reduction technique is then tested numerically in Section 4, and a summary is given in Section 5.

\section{Generalized cross Gramian}

In this section, the cross-Gramian matrix, introduced in [13], is briefly reviewed from the point of view of generalized linear time-invariant (LTI) systems (1).

Fundamental to system-theoretic model reduction are the controllability and observability operators [1], which are given for (1) by the generalized controllability operator $\mathcal{C}: L_{2} \rightarrow \mathbb{R}^{N}$ and the generalized observability operator $\mathcal{O}: \mathbb{R}^{N} \rightarrow L_{2}$ :

$$
\begin{aligned}
\mathcal{C}(u) & :=\int_{0}^{\infty} e^{E^{-1} A t} E^{-1} B u(t) d t, \\
\mathcal{O}\left(x_{0}\right) & :=C e^{E^{-1} A t} E^{-1} x_{0} .
\end{aligned}
$$

The (generalized) cross Gramian ${ }^{2}$ is then defined as a composition of the generalized controllability and observability operators:

$$
W_{X}:=\mathcal{C} \circ \mathcal{O}=\int_{0}^{\infty} e^{E^{-1} A t} E^{-1} B C e^{E^{-1} A t} E^{-1} d t \in \mathbb{R}^{N \times N},
$$

and jointly quantifies controllability and observability of square systems-systems with the same number of inputs and outputs $M=Q$. For linear, square systems with $E=I$, the cross Gramian solves a Sylvester matrix equation [13]; for $E \neq I$, the generalized cross Gramian solves a Sylvester-type equation:

$$
A W_{X} E+E W_{X} A=-B C,
$$

which can be shown using integration-by-parts of (2):

$$
\begin{aligned}
& W_{X}= \int_{0}^{\infty} e^{E^{-1} A t} E^{-1} B C e^{E^{-1} A t} E^{-1} d t \\
&=\left.\left(E^{-1} A\right)^{-1} e^{E^{-1} A t} E^{-1} B C e^{E^{-1} A t} E^{-1}\right|_{0} ^{\infty} \\
& \quad-\left(E^{-1} A\right)^{-1} \int_{0}^{\infty} e^{E^{-1} A t} E^{-1} B C e^{E^{-1} A t}\left(E^{-1} A\right) E^{-1} d t \\
& \Rightarrow A W_{X}=\left.E e^{E^{-1} A t} E^{-1} B C e^{E^{-1} A t} E^{-1}\right|_{0} ^{\infty}-E W_{X} A E^{-1} \\
& \Rightarrow A W_{X} E+E W_{X} A=\left.E e^{E^{-1} A t} E^{-1} \mathrm{BC} e^{E^{-1} A t}\right|_{0} ^{\infty}=-B C .
\end{aligned}
$$

Besides the cross Gramian, the (generalized) controllability Gramian $W_{C}:=\mathcal{C C}^{*}$ and (generalized) observability Gramian $W_{O}:=\mathcal{O}^{*} \mathcal{O}$ are defined accordingly $[37,41]$. For systems with a symmetric Hankel operator $H:=\mathcal{O C}$,

\footnotetext{
${ }^{2}$ Note that the term generalized cross Gramian is used in [38] for cross Gramians of unstable systems.
} 
$H=H^{*}$ [29], for example, all SISO (single-input-single-output) systems, the (generalized) cross Gramian has the property:

$$
W_{X} W_{X}=\mathcal{C O C O}=\mathcal{C}(\mathcal{O C})^{*} \mathcal{O}=\mathrm{CC}^{*} \mathcal{O}^{*} \mathcal{O}=W_{C} W_{O}
$$

Hence, for symmetric systems, either, $W_{X}$ or $\left\{W_{C}, W_{O}\right\}$ can be used interchangeably, if controllability and observability are to be concurrently evaluated. For non-symmetric and especially non-square systems, an approximation to the cross Gramian is defined, based on the column-wise partitioning of the input matrix $B$ and row-wise partitioning of the output matrix $C$ :

$$
B=\left(b_{1} \ldots b_{M}\right), \quad C=\left(c_{1} \ldots c_{Q}\right)^{\top} .
$$

For $\bar{B}:=\sum_{m=1}^{M} b_{m}$ and $\bar{C}:=\sum_{q=1}^{Q} c_{q}^{\top}$, the non-symmetric generalized cross Gramian [20] for (1) is defined as follows:

$$
W_{Z}:=\int_{0}^{\infty} e^{E^{-1} A t} E^{-1} \bar{B} \bar{C} e^{E^{-1} A t} E^{-1} d t,
$$

which is the cross Gramian of the average system $(E, A, \bar{B}, \bar{C})$.

The (non-symmetric) generalized cross Gramian (2) can be computed numerically, for example, using the Hessenberg-Schur algorithm [14], the alternating direction implicit (ADI) algorithm [5-7], or as an empirical cross Gramian [16].

\section{Model reduction}

One of the main numerical applications of the cross Gramian is model (order) reduction, which aims to determine lower order surrogate systems for (1), with respect to the state-space dimension $N:=\operatorname{dim}(x(t))$. The reduced order model (ROM) with $x_{r}: \mathbb{R} \rightarrow \mathbb{R}^{n}, n \ll N$,

$$
\begin{aligned}
E_{r} \dot{x}_{r}(t) & =A_{r} x_{r}(t)+B_{r} u(t), \\
\tilde{y}(t) & =C_{r} x_{r}(t),
\end{aligned}
$$

has a reduced system matrix $A_{r} \in \mathbb{R}^{n \times n}$, a reduced input matrix $B_{r} \in \mathbb{R}^{n \times M}$, a reduced output matrix $C_{r} \in \mathbb{R}^{Q \times n}$ and a reduced mass matrix $E_{r} \in \mathbb{R}^{n \times n}$, such that the reduced system's output $\tilde{y}: \mathbb{R} \rightarrow \mathbb{R}^{Q}$ approximates the full order model's output:

$$
\frac{\|y-\tilde{y}\|}{\|y\|} \ll 1,
$$

in a suitable norm.

Following, the projection-based dominant subspaces model reduction method is extended to exploit the cross Gramian for computation, and the practical computation of the cross-Gramian-based dominant subspaces is discussed. 


\subsection{Projection-based model reduction}

A commonplace approach to construct reduced order models is mapping the statespace trajectory $x(t)$ to a lower dimensional subspace, using a reduction operator $V_{1}: \mathbb{R}^{N} \rightarrow \mathbb{R}^{n}$ and a lifting operator $U_{1}: \mathbb{R}^{n} \rightarrow \mathbb{R}^{N}[33]:$

$$
x_{r}(t):=V_{1} x(t) \quad \rightarrow \quad x(t) \approx U_{1} x_{r}(t) .
$$

In the case of (generalized) linear systems (1), the operators $U_{1} \in \mathbb{R}^{N \times n}$ and $V_{1} \in \mathbb{R}^{n \times N}$, can be directly applied to the system components $A, B, C$, and $E$ to obtain the reduced quantities:

$$
A_{r}:=V_{1} A U_{1}, \quad B_{r}:=V_{1} B, \quad C_{r}:=C U_{1}, \quad E_{r}:=V_{1} E U_{1} .
$$

Hence, the aim is the computation of suitable reducing and lifting operators $U_{1}, V_{1}$, which are typically assumed to be bi-orthogonal $V_{1} U_{1}=I$. The dominant subspaces method, considered in this work, is additionally orthogonal $V_{1}:=U_{1}^{\top}$; thus, the reduction process is a Galerkin projection, which is stability preserving, if the symmetric part of the system matrix $A$ is negative definite, and the mass matrix $E$ positive definite [8, Sec. II.C] (strictly dissipative systems),

$$
A+A^{\top}<0 \wedge E>0 .
$$

This is a generalization of the stability preservation for systems with $E=I$, mentioned in [32, Sec. 4.3]. If a system does not fulfill (6), a stabilization procedure, see for example [4, Sec. 4], can be applied to the ROM.

\subsection{Dominant Subspaces}

The dominant subspaces projection model reduction (DSPMR) is introduced in [32, Sec. 4.3]. The idea behind DSPMR is, instead of balancing controllability and observability Gramians, to combine the associated principal subspaces obtained from approximate system Gramians. This yields a simple model reduction algorithm which is based upon low-rank factors of the controllability and observability Gramians. In [32], a low-rank Cholesky (LR Chol) factor is used, while [23] utilizes singular vectors of a truncated singular value decomposition (tSVD),

$$
\begin{array}{ll}
W_{C} \stackrel{\text { LR Chol }}{\approx} Z_{C} Z_{C}^{\top}, & W_{O} \stackrel{\text { LR Chol }}{\approx} Z_{O} Z_{O}^{\top}, \\
W_{C} \stackrel{\mathrm{tSVD}}{\approx} U_{C} D_{C} U_{C}^{\top}, & W_{O} \stackrel{\mathrm{tSVD}}{\approx} U_{O} D_{O} U_{O}^{\top} .
\end{array}
$$

The controllability and observability subspaces encoded in the matrix factors are now conjoined and orthogonalized, by either a rank-revealing SVD ([32]) or a rankrevealing QR decomposition $([23,24])$. Either the left singular vectors $U$ or the $Q$ factor can be taken as Galerkin projections, respectively:

$$
\begin{aligned}
\mathrm{QR} & \stackrel{\mathrm{QR}}{=}\left[\begin{array}{ll}
U_{C} & U_{O}
\end{array}\right] \rightarrow U_{1}:=Q, \\
\mathrm{UDV}^{\top} & \stackrel{\mathrm{SVD}}{=}\left[\begin{array}{ll}
U_{C} & U_{O}
\end{array}\right] \rightarrow U_{1}:=U,
\end{aligned}
$$


see also [2, Sec 2.1.7]. Compared to POD (proper orthogonal decomposition) [1, Ch. 9.1], which in this context is equivalent to using solely the controllability subspace (basis) $U_{C}$ as a Galerkin projection, DSPMR incorporates controllability and observability information. Yet, in comparison to balanced POD [30, 36, 45], the truncated controllability and observability subspaces $U_{C}, U_{O}$ are not balanced, but directly concatenated.

An extension to the DSPMR method is also proposed in [32], called refined dominant subspace projection model reduction. The eponymous refinement is given by weighting factors $\omega_{C}, \omega_{O}>0$ for the controllability and observability subspace bases respectively. The weighting factors are selected as the Frobenius norm of the respective low-rank factors, $\omega_{C}:=\left\|Z_{C}\right\|_{\mathrm{F}}^{-1}$ and $\omega_{O}:=\left\|Z_{O}\right\|_{\mathrm{F}}^{-1}$, yielding:

$$
\begin{aligned}
& \mathrm{QR} \stackrel{\mathrm{QR}}{=}\left[\left(\omega_{C} Z_{C}\right)\left(\omega_{O} Z_{O}\right)\right] \rightarrow U_{1}:=Q, \\
& \mathrm{UDV}^{\top} \stackrel{\mathrm{SVD}}{=}\left[\left(\omega_{C} Z_{C}\right)\left(\omega_{O} Z_{O}\right)\right] \rightarrow U_{1}:=U \text {. }
\end{aligned}
$$

Obviously, this is only sensible for the Cholesky factor variant, as the norm of the (orthonormal) singular vectors is one.

The weighting normalizes the system Gramian factors. This normalization equilibrates the influence of controllability ( $W_{C}$ depends only on $\{A, B\}$ ) and observability ( $W_{O}$ depends only on $\{A, C\}$ ), which may be skewed, i.e., due to different scaling of $B$ and $C$. A similar idea for combining weighted subspaces is also used in the cotangent lift method from [31].

\subsection{Cross-Gramian-Based Dominant Subspaces}

Instead of the controllability and observability Gramians, also the cross Gramian can be used to obtain a dominant subspace projection. A truncated SVD of the cross Gramian (based on a pre-selected rank or approximation error),

$$
W_{X} \stackrel{\mathrm{tSVD}}{=} U_{X} D_{X} V_{X}^{\top}
$$

produces left and right singular vectors aggregated in matrices $U_{X}$ and $V_{X}$, which induce subspaces associated to controllability $\left(U_{X}\right)$ and observability $\left(V_{X}\right)$ of the underlying system $(A, B, C, E)[47$, Sec. B].

In [40, Sec. 4.3], it is noted, that the sole use of either, $U_{X}$ or $V_{X}$, as a Galerkin projection, will largely omit observability or controllability information respectively. Hence, both subspaces should be incorporated in the reducing and lifting operator. Balanced truncation, for example, determines a suitable Petrov-Galerkin projection, ${ }^{3}$ where $U_{1} \neq V_{1}$, by simultaneous diagonalization of the controllability and observability Gramians, while approximate balancing applies the left and right singular vectors of the cross Gramian as oblique projections directly [34].

\footnotetext{
${ }^{3}$ Balanced truncation yields a Galerkin projection for state-space symmetric systems, $A=A^{\top}, B=C^{\top}, E=E^{\top}[9]$.
} 
For the proposed variant of the dominant subspace method (for an algorithmic description see Section 3.3.1), the left and right singular vectors are conjoined as before, but also scaled column-wise by the associated singular values:

$$
\left[\left(U_{X} D_{X}\right)\left(V_{X} D_{X}\right)\right] \stackrel{\mathrm{SVD}}{=} U_{C O} D_{C O} V_{C O}^{\top} \rightarrow U_{1}:=U_{C O}
$$

Here, the singular values are used to scale the singular vectors, since the majorization property [40, Remark 2.1] relates the singular values of the cross Gramian with the (absolute value of the) cross Gramian's eigenvalues, which in turn are equal to the Hankel singular values of a symmetric system (3). So, instead of normalizing the controllability and observability subspaces (as a whole), as in refined DSPMR, based on the common controllability-observability measure (the singular values of the cross Gramian), the vectors spanning the compound subspace are scaled individually. Here explicitly a rank-revealing SVD is used, instead of a QR decomposition, as the singular values $D_{C O}$ will be used for an error indicator in Section 3.4. An advantage of the cross-Gramian-based dominant subspace projection method is this common measure of minimality [12]: The singular values $\sigma_{i}=D_{C O, i i}$ associated jointly to the "controllability" and "observability" subspaces.

\subsubsection{Algorithmic computation}

The computation of the proposed cross-Gramian-based dominant subspace projection, as well as the classic dominant subspace projection consists of two phases: first, the computation of the system Gramians, either the cross Gramian or the controllability and observability Gramians; second, the assembly of the reducing (and lifting) operator.

For large-scale systems, the computation of dense system Gramians, which are of dimension $N \times N$, may be infeasible or at least inefficient. To this end, low-rank representations of the Gramians can be computed, for the cross Gramian, in example by the implicitly restarted Arnoldi algorithm [40], the factorized iteration [3], a factored ADI [5] or a low-rank empirical cross Gramian [19].

Overall, the cross-Gramian-based dominant subspace algorithm is summarized by the following:

1. Compute (low-rank) cross Gramian:

(a) As solution to a matrix equation: $A W_{X} E+E W_{X} A=-B C$,

(b) or by quadrature: $W_{X}=\int_{0}^{\infty} e^{E^{-1} A t} E^{-1} B C e^{E^{-1} A t} E^{-1} d t$.

2. Compute (truncated) SVD of the cross Gramian:

$$
U_{X} D_{X} V_{X}^{\top} \stackrel{\mathrm{tSVD}}{=} W_{X} .
$$

3. Compute (rank-revealing) SVD of conjoined and weighted left and right singular vectors:

$$
U_{1} D_{1} V_{1} \stackrel{\text { SVD }}{=}\left[\left(U_{X} D_{X}\right)\left(V_{X} D_{X}\right)\right] .
$$


4. Apply left singular vectors to system matrices following (5):

$$
A_{r}:=U_{1}^{\top} A U_{1}, \quad B_{r}:=U_{1}^{\top} B, \quad C_{r}:=C U_{1}, \quad E_{r}:=U_{1}^{\top} E U_{1} .
$$

The Galerkin projection $U_{1}$ is the cross-Gramian-based dominant subspace projection. In principle, a similar procedure can be conducted using controllability and observability Gramians, yet it is not immediately clear if the SVD of the (weighted) conjoined singular vectors $\left[\left(U_{C} D_{C}\right)\left(U_{O} D_{O}\right)\right]$ yields an equally useful measure. The efficiency of computing a low-rank approximation of $W_{X}$ depends on the rank of BC and the symmetry of $A$. Usually, this means, the more (linearly) independent inputs and outputs a system has, and the less symmetric a system matrix is, the higher the rank of the approximated cross Gramian.

\subsection{Error indicator}

In this section, an error indicator for the cross-Gramian-based dominant subspace method is developed. Previous works, such as [35, 39, 44, 46], already introduced error bounds for the Hardy $\mathrm{H}_{2}$-norm. Here, an $\mathrm{H}_{2}$-error indicator of simple structure using time-domain quantities is proposed, which is loosely related to the simplified balanced gains approach from [11]. The $H_{2}$-norm is particularly interesting, since an error estimation has relevance for the frequency-domain and the time-domain [43, Ch. 2], and it also describes the energy ( $L_{2}$-norm) of the system's impulse response. Before this error indicator is derived, a straightforward property of the matrix exponential is presented.

Lemma 1 Given matrices $A \in \mathbb{R}^{N \times N}$ and $U \in \mathbb{R}^{N \times n}, n \leq N$, the following holds:

$$
U e^{U A U^{\top}} U^{\top}=U U^{\top} e^{A U U^{\top}}=e^{U U^{\top} A} U U^{\top} .
$$

Proof The proof is a trivial consequence on the associativity of the matrix product.

$$
\begin{aligned}
U e^{U^{\top} A U} U^{\top} & =U\left(\sum_{k=0}^{\infty} \frac{1}{k !}\left(U^{\top} A U\right)^{k}\right) U^{\top} \\
& =U\left(I+\left(U^{\top} A U\right)+\frac{1}{2}\left(U^{\top} A U\right)\left(U^{\top} A U\right)+\ldots\right) U^{\top} \\
& =U U^{\top}\left(I+A U U^{\top}+\frac{1}{2} A U U^{\top} A U U^{\top}+\ldots\right) \\
& =U U^{\top} e^{A U U^{\top}} .
\end{aligned}
$$

Next, the error indicator is constructed, which is derived from the $L_{2}$-norm of the impulse response error system, and we assume, for ease of exposition but without loss of generality, $E=I$ :

$$
\begin{aligned}
\left(\begin{array}{c}
\dot{x}(t) \\
\dot{x}_{r}(t)
\end{array}\right) & =\left(\begin{array}{cc}
A & 0 \\
0 & A_{r}
\end{array}\right)\left(\begin{array}{c}
x(t) \\
x_{r}(t)
\end{array}\right)+\left(\begin{array}{c}
B \\
B_{r}
\end{array}\right) u(t) \\
y_{e}(t) & =\left(\begin{array}{ll}
C & -C_{r}
\end{array}\right)\left(\begin{array}{c}
x(t) \\
x_{r}(t)
\end{array}\right) .
\end{aligned}
$$


We consider only SISO systems for this error indicator and unit impulse (Dirac impulse) inputs $u(t) \equiv \delta(t)$, defined by the properties:

$$
\int \delta(t) d t=1, \quad \delta(t \neq 0)=0 .
$$

First, the $\mathrm{H}_{2}$-norm of the error system, in impulse response form, is transformed in a manner so that Lemma 1 can be applied. Note that the error system of a SISO system is also a SISO system with a scalar and thus symmetric impulse response:

$$
\begin{aligned}
\left\|y_{e}\right\|_{L_{2}}^{2}= & \operatorname{tr}\left(\int_{0}^{\infty}\left(\left(C-C_{r}\right)\left(\begin{array}{cc}
e^{\mathrm{At}} & 0 \\
0 & e^{A_{r} t}
\end{array}\right)\left(\begin{array}{c}
B \\
B_{r}
\end{array}\right)\right)^{2} d t\right) \\
= & \operatorname{tr}\left(\int_{0}^{\infty}\left(C e^{\mathrm{At}} B-C_{r} e^{A_{r} t} B_{r}\right)^{2} d t\right) \\
= & \operatorname{tr}\left(C \int_{0}^{\infty} e^{\mathrm{At}} \mathrm{BC} e^{\mathrm{At}}-e^{\mathrm{At}} \mathrm{BC}_{r} e^{A_{r} t} U_{1}^{\top}\right. \\
& \left.-U_{1} e^{A_{r} t} B_{r} C e^{\mathrm{At}}+U_{1} e^{A_{r} t} B_{r} C_{r} e^{A_{r} t} U_{1}^{\top} d t B\right),
\end{aligned}
$$

applying the definition of the reduced quantities (5), and subsequently the result of Lemma 1, gives the following:

$$
\begin{aligned}
\left\|y_{e}\right\|_{L_{2}}^{2}=\operatorname{tr}\left(C \int_{0}^{\infty} e^{A t} B C e^{A t}\right. & -e^{A t} B C e^{U_{1} U_{1}^{\top} A t} U_{1} U_{1}^{\top} \\
& -U_{1} U_{1}^{\top} e^{A U_{1} U_{1}^{\top} t} B C e^{A t} \\
& \left.+U_{1} U_{1}^{\top} e^{A U_{1} U_{1}^{\top} t} B C e^{U_{1} U_{1}^{\top} A} U_{1} U_{1}^{\top} d t B\right) .
\end{aligned}
$$

The next step is approximating the matrix exponentials $e^{\mathrm{AU}_{1} U_{1}^{\top} t}$ and $e^{U_{1} U_{1}^{\top} \text { At }}$ by the homogeneous system's solution operator,

$$
e^{A U_{1} U_{1}^{\top} t} \approx e^{A t}, \quad e^{U_{1} U_{1}^{\top} A t} \approx e^{A t},
$$

which allows to factor the previous representation to the following:

$$
\left\|y_{e}\right\|_{L_{2}}^{2} \approx \operatorname{tr}\left(C \int_{0}^{\infty}\left(I-U_{1} U_{1}^{\top}\right)\left(e^{A t} B C e^{A t}\right)\left(I-U_{1} U_{1}^{\top}\right) d t B\right) .
$$

Now, we move the projection error terms $\left(I-U_{1} U_{1}^{\top}\right)$ out of the integral, identify the resulting expression with the cross Gramian $W_{X}$, and exploit the cyclic permutability of the trace argument:

$$
\begin{aligned}
\left\|y_{e}\right\|_{L_{2}}^{2} & \approx \operatorname{tr}\left(C\left(I-U_{1} U_{1}^{\top}\right) \int_{0}^{\infty} e^{A t} B C e^{A t} d t\left(I-U_{1} U_{1}^{\top}\right) B\right) \\
& =\operatorname{tr}\left(\left(I-U_{1} U_{1}^{\top}\right) W_{X}\left(I-U_{1} U_{1}^{\top}\right) B C\right) .
\end{aligned}
$$

The (full) SVD of the cross Gramian is given by adding to its truncated SVD, $W_{X} \stackrel{\text { tSVD }}{=} U_{X} D_{X} V_{X}^{\top}$, (the SVD of) its truncated remainder:

$$
W_{X} \stackrel{\text { SVD }}{=} U_{X} D_{X} V_{X}^{\top}+U_{2} D_{2} V_{2}^{\top} \text {. }
$$


Together with an observation on the truncated SVD's singular vectors:

$$
\begin{aligned}
& U_{X}=\left[\begin{array}{ll}
\left(U_{X} D_{X}\right) & \left(V_{X} D_{X}\right)
\end{array}\right]\left[\begin{array}{c}
D_{X}^{-1} \\
0
\end{array}\right]=U_{1} D_{1} V_{1}^{\top}\left[\begin{array}{c}
D_{X}^{-1} \\
0
\end{array}\right], \\
& V_{X}=\left[\begin{array}{ll}
\left(U_{X} D_{X}\right) & \left(V_{X} D_{X}\right)
\end{array}\right]\left[\begin{array}{c}
0 \\
D_{X}^{-1}
\end{array}\right]=U_{1} D_{1} V_{1}^{\top}\left[\begin{array}{c}
0 \\
D_{X}^{-1}
\end{array}\right],
\end{aligned}
$$

the following simplification entails:

$$
\begin{aligned}
& \left(I-U_{1} U_{1}^{\top}\right) W_{X}\left(I-U_{1} U_{1}^{\top}\right) \\
= & \left(I-U_{1} U_{1}^{\top}\right)\left(U_{X} D_{X} V_{X}^{\top}+U_{2} D_{2} V_{2}^{\top}\right)\left(I-U_{1} U_{1}^{\top}\right) \\
= & \left(I-U_{1} U_{1}^{\top}\right)\left(U_{2} D_{2} V_{2}^{\top}\right)\left(I-U_{1} U_{1}^{\top}\right) .
\end{aligned}
$$

Next, the VON NEUMANN's trace inequality [26], which assumes (without loss of generality) descendingly ordered singular values $\sigma_{k}(\cdot) \geq \sigma_{k+1}(\cdot)$ is applied, followed by the Cauchy-Schwarz inequality (with $\sigma(\cdot)$ being the vector of singular values):

$$
\begin{aligned}
\left\|y_{e}\right\|_{L_{2}}^{2} & \approx \operatorname{tr}\left(\left(I-U_{1} U_{1}^{\top}\right)\left(U_{2} D_{2} V_{2}\right)\left(I-U_{1} U_{1}^{\top}\right) B C\right) \\
& \leq \sum_{k=1}^{N} \sigma_{k}\left(D_{2}\right) \sigma_{k}(B C)=\left\langle\sigma\left(D_{2}\right), \sigma(B C)\right\rangle \\
& \leq\left\|\sigma\left(D_{2}\right)\right\|_{2}\|\sigma(B C)\|_{2}=\left\|D_{2}\right\|_{F}\|B C\|_{F} .
\end{aligned}
$$

Since the singular values of $D_{2}$ correspond to the truncated tail of the cross Gramian's singular values, and BC is of rank one, due to the SISO nature of the system, we obtain the following:

$$
\left\|y_{e}\right\|_{L_{2}}^{2} \lesssim\|B C\|_{2} \sqrt{\sum_{k=n+1}^{N} \sigma_{k}^{2}\left(W_{X}\right)} .
$$

Note that $\|B C\|_{2}=\|B\|_{2}\|C\|_{2}$, as $B$ and $C^{\top}$ are column vectors. Overall, this derivation yields the following error indicator:

Error Indicator The $L_{2}$ impulse response model reduction error for a cross-Gramianbased dominant subspaces reduced order model is approximated by the following:

$$
\|y-\tilde{y}\|_{L_{2}} \lesssim \sqrt{\|B\|_{2}\|C\|_{2} \sqrt{\sum_{k=n+1}^{N} \sigma_{k}^{2}\left(W_{X}\right)} .}
$$

Remark 1 Using the Cauchy-Schwarz inequality as in [15], this impulse response error indicator can be extended to squarely integrable inputs $u \in L_{2}$.

The error indicator directly extends to symmetric MIMO systems, utilizing $C e^{A} B=\left(C e^{A} B\right)^{\top}$ and noting $\|B C\|_{2} \leq\|B\|_{2}\|C\|_{2}$. For (just) square MIMO 
systems, the derivation would have to be adapted, leading to an error indicator that is not based on the cross Gramian's singular values any more, which is essential to the practical computation detailed in Section 3.5. Alternatively, it extends to any MIMO systems by either using the averaged system $\left(A, \sum_{i=1}^{M} b_{i}, \sum_{j=1}^{Q} c_{j}^{\top}\right)$ associated to the non-symmetric cross Gramian (4) [20], or, selecting a SISO sub-system $\left(A, b_{k}, c_{\ell}^{\top}\right)$, for example based on the following:

$$
(k, \ell)=\underset{i, j}{\arg \max }\left\langle\left|b_{i}\right|,\left|c_{j}\right|\right\rangle .
$$

Furthermore, the error indicator holds also for systems with $E \neq I, E>0$ :

$$
\|y-\tilde{y}\|_{L_{2}} \lesssim \sqrt{\left\|E^{-1} B\right\|_{2}\|C\|_{2} \sqrt{\sum_{k=n+1}^{N} \sigma_{k}^{2}\left(W_{X}\right)}}
$$

which follows from: $E \dot{x}(t)=A x(t)+B u(t) \Rightarrow \dot{x}(t)=E^{-1} A x(t)+E^{-1} B u(t)$.

\subsection{Fused computation}

Even for moderately sized systems, the computation of the (cross) Gramian's singular vectors may be a computationally challenging task. ${ }^{4}$ To compute the dominant subspace projections from the cross Gramian, or the controllability and observability Gramians, the hierarchical approximate proper orthogonal decomposition (HAPOD) [18] is used.

The HAPOD enables a swift computation of left singular vectors of arbitrary partitioned data sets (matrices), based on a selected projection error (on the input data) $\varepsilon>0$ and a tree hierarchy with the data (Gramian) partitions as leafs. The tree hierarchy utilized for the experiments in this work is given by a combination of special topologies discussed in [18], the incremental HAPOD (maximally unbalanced binary tree) and the distributed HAPOD (star). Two incremental HAPODs are performed for the Gramian partitions respectively, and subsequently, a distributed HAPOD of the resulting singular vectors from both sub-trees yield the dominant subspace projection. Figure 1 illustrates the overall HAPOD tree.

Since the HAPOD computes only left singular vectors, but the right singular vectors of the cross Gramian are also needed, the HAPOD of the cross Gramian (left singular vectors) and the transposed cross Gramian (right singular vectors) is computed. In the following numerical examples, the (full-order) empirical linear cross Gramian [16, Sec. 3.1.3] is used, as in-memory storage of the Gramian(s) is possible. For settings, where only parts of the cross Gramian can be kept in memory, the low-rank empirical cross Gramian [19] for the left singular vectors, and the low-rank empirical cross Gramian of the adjoint system for the right singular vectors, can be utilized,

\footnotetext{
${ }^{4}$ For the presented numerical examples the SVDs of system Gramians comprises the dominant fraction of computation time.
} 


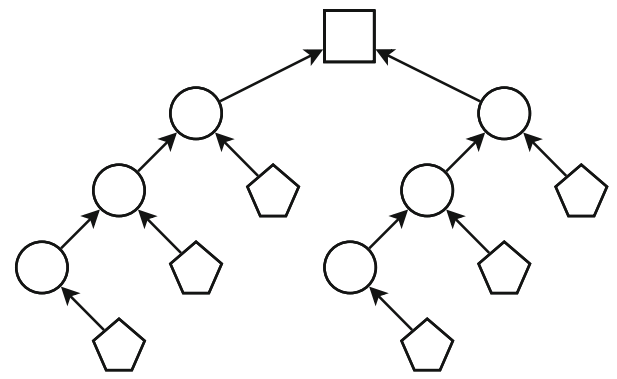

Fig. 1 HAPOD tree topology for the cross-Gramian-based dominant subspaces method. Pentagons symbolize partitions of the cross Gramian (left) and the adjoint cross Gramian (right), respectively. Circles mark sub-PODs, while a square represents the root-POD returning the overall (HA)POD described in Section 3.5. In the context at hand, PODs correspond to SVDs

since the cross Gramian of the adjoint system is equal to the system's transposed cross Gramian as follows:

$$
\begin{aligned}
\widetilde{W}_{X} & :=\int_{0}^{\infty} e^{E^{-\top} A^{\top} t} E^{-\top} C^{\top} B^{\top} e^{E^{-\top} A^{\top} t} E^{-\top} d t \\
& =\int_{0}^{\infty}\left(\mathrm{CE}^{-1} e^{A E^{-1} t}\right)^{\top}\left(E^{-1} e^{A E^{-1} t} B\right)^{\top} d t \\
\stackrel{\text { Lemma }}{=} \int_{0}^{\infty}\left(C e^{E^{-1} A t} E^{-1}\right)^{\top}\left(e^{E^{-1} A t} E^{-1} B\right)^{\top} d t & \\
& =\int_{0}^{\infty}\left(e^{E^{-1} \mathrm{At}} E^{-1} \mathrm{BC} e^{E^{-1} A t} E^{-1}\right)^{\top} d t=W_{X}^{\top} .
\end{aligned}
$$

Since a projection-error-driven SVD method is used, the following error bound holds, ${ }^{5}$ for a given projection error $\varepsilon>0$ :

$$
\sum_{k=1}^{N}\left\|\left(I-U_{1} U_{1}^{\top}\right) W_{X, * k}\right\|^{2}=\sum_{k=n+1}^{N} \sigma_{k}^{2}\left(W_{X}\right) \leq \varepsilon^{2} .
$$

This means the error indicator (9) can be bounded using the prescribed cross Gramian's projection error as follows:

$$
\|y-\tilde{y}\|_{L_{2}} \lesssim \sqrt{\|B\|_{2}\|C\|_{2} \sqrt{\sum_{k=n+1}^{N} \sigma_{k}^{2}\left(W_{X}\right)}} \leq \sqrt{\varepsilon\|B\|_{2}\|C\|_{2}},
$$

thus making it an a-priori error indicator. This approximate error prediction for a given projection error $\varepsilon$ and the Euclidean norms (spectral norms) of the input and output operators, without computing any system Gramians, is the main advantage of this method. The tightness of this error indicator is evaluated in the following numerical results.

\footnotetext{
${ }^{5}$ This is shown for the HAPOD in [18]. Specifically, the mean $L_{2}$ projection error, $\frac{1}{N} \sum_{k=1}^{N}\left\|\left(I-U_{1} U_{1}^{\top}\right) W_{X, * k}\right\|^{2} \leq \varepsilon^{2}$, is bounded by the HAPOD, which has to be taken into account for the practical computation.
} 


\section{Numerical results}

Following, two numerical examples are presented to illustrate the previous findings. These numerical experiments are conducted using MATLAB 2018a [25]. The system Gramians needed for the dominant subspace methods, the controllability and observability Gramian for plain and refined DSPMR as well as balanced truncation, and the cross Gramian for the cross-Gramian-based dominant subspaces, are computed as empirical Gramians [16] using emgr-empirical Gramian framework in version 5.7 [17]. All simulated trajectories for the construction of these empirical dominant subspaces are computed using the implicit Euler method, and the HAPOD is computed via [21].

\subsection{FOM benchmark}

The first numerical example compares the cross-Gramian-based dominant subspace method with the classic unrefined and refined dominant subspace method ${ }^{6}$ as well as (empirical) balanced truncation ${ }^{7}$ for the "FOM" example in [32], which is also part of the SLICOT Benchmark Collection [10]. This linear SISO system (with $E=I$ ) of the structure:

$$
\begin{aligned}
\dot{x}(t) & =A x(t)+B u(t), \\
y(t) & =C x(t),
\end{aligned}
$$

is of order $N=1006$, and the system components are given by the following:

$$
\begin{aligned}
& A_{1}=\left(\begin{array}{cc}
-1 & 100 \\
-100 & -1
\end{array}\right), \quad A_{2}=\left(\begin{array}{cc}
-1 & 200 \\
-200 & -1
\end{array}\right), \quad A_{3}=\left(\begin{array}{cc}
-1 & 400 \\
-400 & -1
\end{array}\right), \\
& A_{4}=\left(\begin{array}{llll}
-1 & & & \\
& -2 & & \\
& & \ddots & \\
& & & -1000
\end{array}\right), \quad A=\left(\begin{array}{llll}
A_{1} & & & \\
& A_{2} & & \\
& & A_{3} & \\
& & & A_{4}
\end{array}\right) \text {, } \\
& C=\left(\begin{array}{ll}
C_{1} C_{2}
\end{array}\right), \quad C_{1}=(10 \ldots 10) \in \mathbb{R}^{6}, \quad C_{2}=(1 \ldots 1) \in \mathbb{R}^{1000}, \\
& B=C^{\top} \text {. }
\end{aligned}
$$

The empirical Gramians are constructed using random binary input, and the reduced systems are tested with impulse input, to evaluate the error indicator.

In Fig. 2, the (empirical) balanced truncation, the (empirical) dominant subspaces method, the (empirical) refined dominant subspaces method, the (empirical) crossGramian-based dominant subspaces method, the predicted error (11), and the error indicator (10) are compared, for a given projection error $\varepsilon \in\left\{10^{-3}, \ldots, 10^{-12}\right\}$ of

\footnotetext{
${ }^{6}$ The refined DSPMR method is computed with weighting coefficients $\omega_{C}=\frac{\left\|Z_{O}\right\|_{F}}{\left\|Z_{C}\right\|_{F}}$, $\omega_{O}=1$ of the controllability and observability factors respectively for numerical reasons.

${ }^{7}$ In the numerical experiments at hand, low-rank Gramians are balanced, whereas the rank is determined by the projection error of the POD compression of the empirical controllability and observability Gramian. In this sense, this method is related to balanced POD [36].
} 


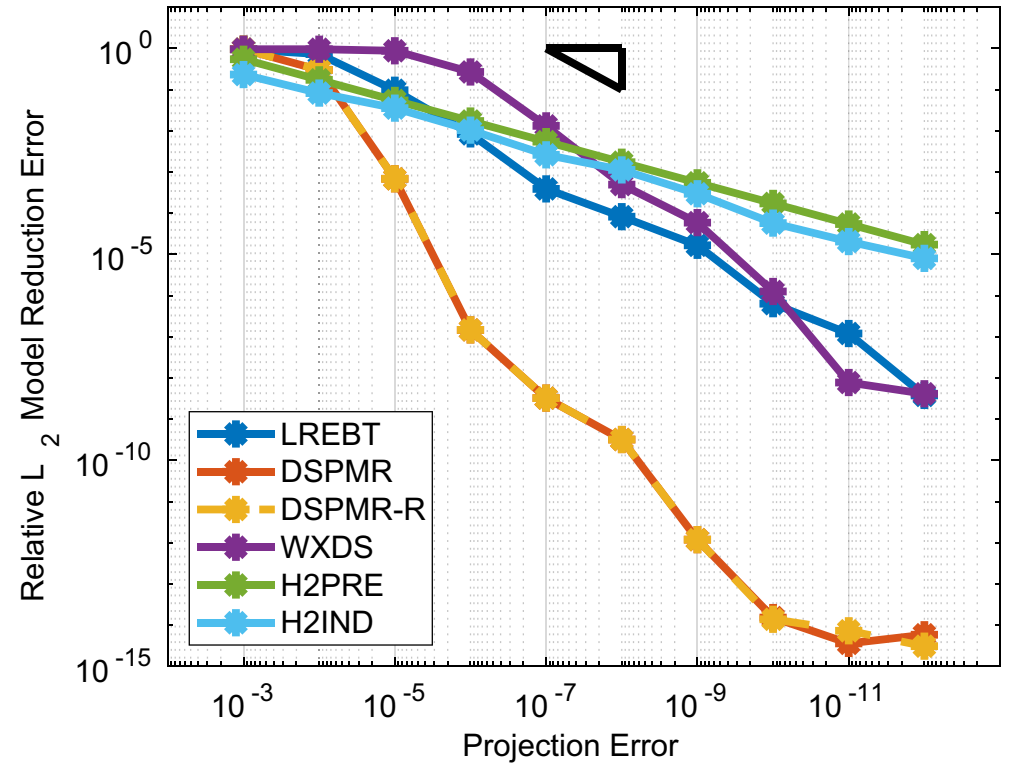

(a) Cross Gramian projection error versus $L_{2}$ model reduction error.

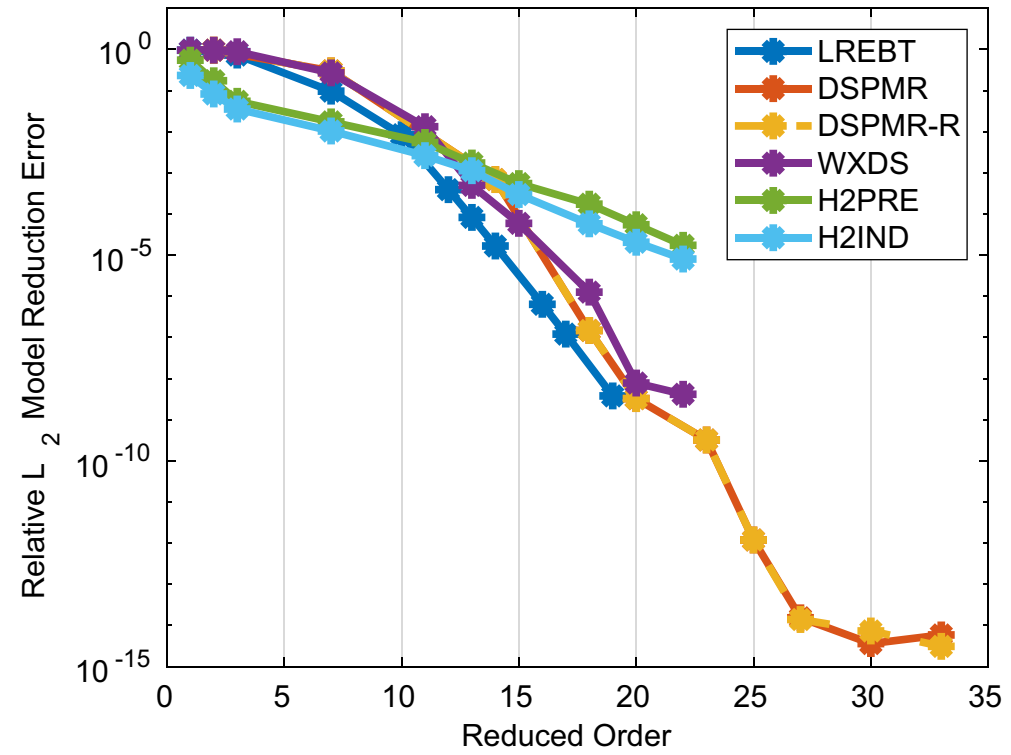

(b) Reduced order versus $L_{2}$ model reduction error.

Fig. 2 Model reduction error of the FOM benchmark example from Section 4.1 for low-rank empirical balanced truncation (LREBT), dominant subspaces (DSPMR), refined dominant subspaces (DSPMR-R), cross-Gramian-based dominant subspaces (WXDS), the predicted $\mathrm{H}_{2}$-error (H2PRE), and the $\mathrm{H}_{2}$-error indicator (H2IND) 
the utilized empirical cross Gramians. The same projection error is selected for the controllability and observability Gramians used by the unrefined DSPMR, refined DSPMR, and low-rank empirical balanced truncation.

In Fig. 2a, the prescribed projection error of the respective Gramians is plotted against the resulting relative $L_{2}$ model reduction error. For a given projection error, the refined DSPMR and DSPMR-R method produce the lowest model reduction error, and low-rank balanced truncation the largest, while the proposed cross-Gramianbased dominant subspace method is in-between. The error indicator overestimates the error for larger and underestimates for smaller projection errors, the predicted error is reasonably close to the error indicator. Note that the error indicator is the square root of the projection error scaled by a constant; hence, it appears as a line in the log-log plot.

Figure $2 \mathrm{~b}$ depicts the resulting reduced order of the tested methods against the model reduction error. Balanced truncation produces the smallest, and DSPMR, DSPMR-R the largest reduced models, again the cross-Gramian-based method is in-between. These results follow intuitions that DSPMR produces the most accurate, but largest subspaces, while balanced truncation may have a smaller, and hence less accurate subspaces. Hence, the cross-Gramian-based dominant subspace method appears as a compromise. The error indicator is rather coarse, which is due to its simple structure.

\subsection{Convection benchmark}

The second numerical example evaluates the convection benchmark [42, Convection $]^{8}$ from the Oberwolfach Benchmark Collection [28]. This is a two-dimensional computational fluid dynamics application of thermal flow modeled by a convectiondiffusion partial differential equation:

$$
\frac{\partial T}{\partial t}=\kappa \nabla^{2} T-v \nabla T+\dot{q}
$$

with the solution temperature $T(x, t)$, the thermal conductivity $\kappa$, the fluid speed $v$ of fixed direction, and the heat generation rate $\dot{q}$. The model is discretized in space using the finite element method, yielding a generalized linear system (1) of order $N=9669 \approx 10^{4}$, a single input $M=1$, five outputs $Q=5$, and $E \neq I$. For a more detailed description of this benchmark, see [28] and references therein. This model is tested in two variants: First, in a symmetric setting with zero flow speed $v=0$, and second, in a non-normal setting with a flow speed $v=0.5$. Due to the (nonsquare) MIMO nature of the system, we use the average system (see (4)) for the error indicator computation.

This set of experiments is organized in the same manner as Section 4.1, but conducted for the prescribed projection errors $\varepsilon \in\left\{10^{-2}, \ldots, 10^{-8}\right\}$. As indicated in Section 3.4, the average system (averaged over outputs) $\bar{C}:=\sum_{q=1}^{Q} c_{q}^{\top}$ is used for the computation of the error indicator. The resulting reduced order models are tested with impulse input $u(t)=\delta(t)$.

\footnotetext{
${ }^{8}$ http://modelreduction.org/index.php/Convection
} 


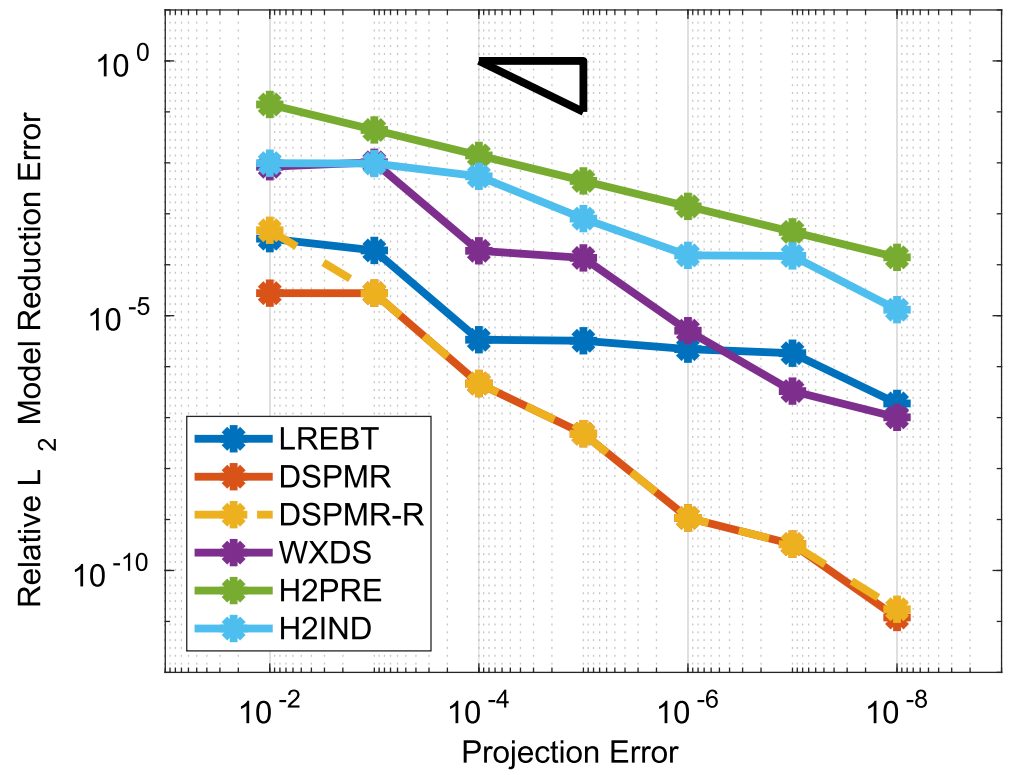

(a) Cross Gramian projection error versus $L_{2}$ model reduction error.

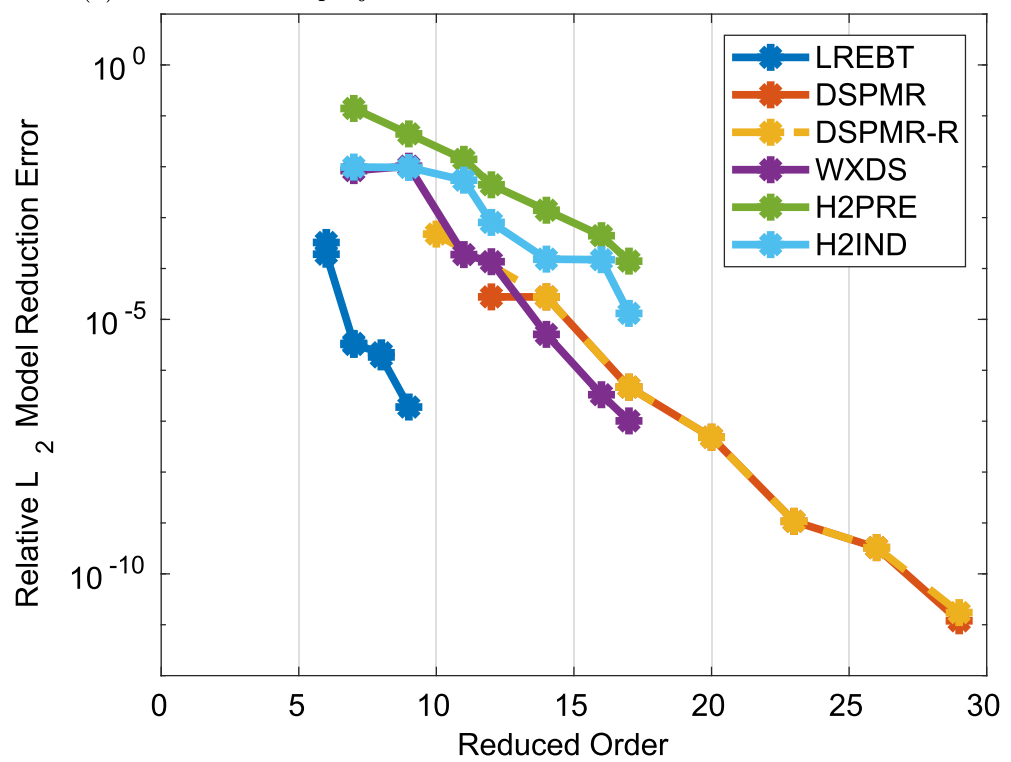

(b) Reduced order versus $L_{2}$ model reduction error.

Fig. 3 Model reduction error of the symmetric convection benchmark from Section 4.2 for low-rank empirical balanced truncation (LREBT), dominant subspaces (DSPMR), refined dominant subspaces (DSPMR-R), cross-Gramian-based dominant subspaces (WXDS), the predicted $\mathrm{H}_{2}$-error (H2PRE), and the $\mathrm{H}_{2}$-error indicator (H2IND) 


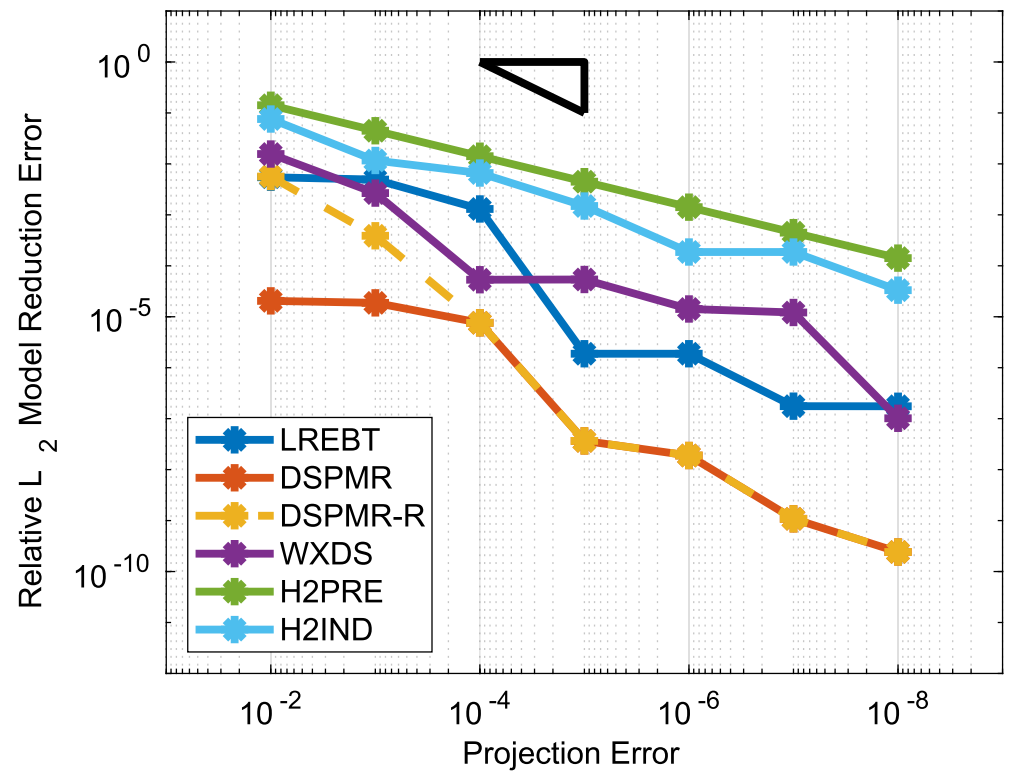

(a) Cross Gramian projection error versus $L_{2}$ model reduction error.

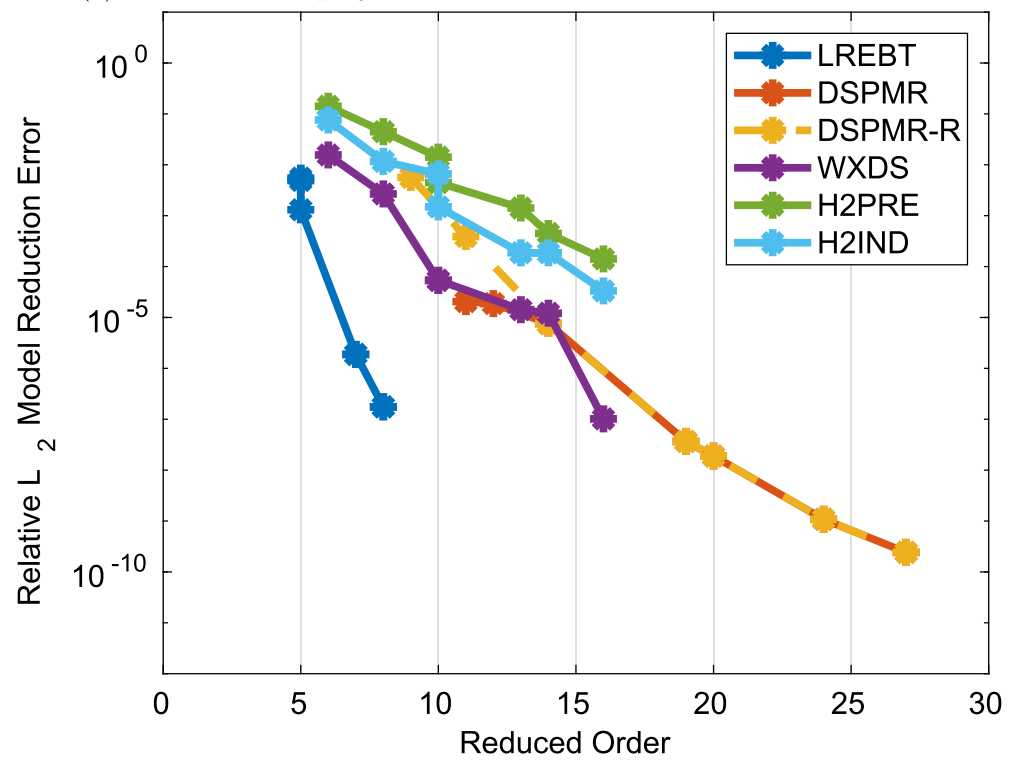

(b) Reduced order versus $L_{2}$ model reduction error.

Fig. 4 Model reduction error of the non-normal convection benchmark from Section 4.2 for low-rank balanced truncation (LREBT), dominant subspaces (DSPMR), refined dominant subspaces (DSPMR-R), cross-Gramian-based dominant subspaces (WXDS), the predicted $\mathrm{H}_{2}$-error (H2PRE) and the $\mathrm{H}_{2}$-error indicator (H2IND) 


\subsubsection{Symmetric variant}

The experimental results of the symmetric variant $(v=0)$ are depicted in Fig. 3. In Fig. 3a, the prescribed projection error for the (empirical) system Gramians versus the resulting relative $L_{2}$ model reduction error is plotted. As in Section 4.1, the DSPMR method produces the ROMs with the lowest model reduction error. The refined DSPMR exhibits slightly larger model reduction errors for small projection errors; otherwise, it is following the plain DSPMR method. Reduced systems from (empirical) balanced truncation and the (empirical) cross-Gramian-based dominant subspace method result in similar errors, while the error indicator behaves like an upper bound to the cross-Gramian-based model reduction error.

Figure $3 \mathrm{~b}$ shows the model reduction error for the reduced orders resulting from the prescribed projection error. Balanced truncation achieves the smallest and DSPMR the largest reduced models, the cross-Gramian-based dominant subspace method reduced order model dimension lies in-between, and the error indicator shows a similar behavior as the latter.

\subsubsection{Non-normal variant}

The experimental results of the non-normal variant $(v=0.5)$ are presented in Fig. 4. Overall, the plots Fig. 4a and Fig. 4b are similar to the symmetric variant, with a reasonably close predicted error, which is equal for symmetric and non-normal benchmark variants. Yet, in case of the non-normal benchmark variant, the error indicator is not as tight, compared to the symmetric variant.

\section{Summary}

In this work, we revisited the dominant subspaces projection model reduction method, and presented a variant based on the cross Gramian matrix for generalized linear systems. This model reduction algorithm requires only a single low-rank (HAPOD) decomposition of the cross Gramian, and provides an a-priori error indicator. Overall, the cross-Gramian-based dominant subspaces technique is a systemtheoretic model reduction method with a simple formulation, efficient computation, conditional stability preservation, and error quantification. The error indicator for the cross-Gramian-based dominant subspace model reduction could be enhanced, for example, by fitting the known singular values exponentially and incorporate such an empirical decay rate. The applicability of this method to control-affine nonlinear systems will be subject of future work, which is in principal possible due to the utilized empirical Gramian computation leading to empirical dominant subspaces.

Acknowledgments This work is dedicated to the late Thilo Penzl, who wrote the preprint version of [32] 20 years (at this time of writing) ago, in 1999, and, moreover, 2019 marks the year of his 20th death anniversary. Thilo Penzl died December 17, 1999, but his work and ideas inspire researchers in model reduction and matrix equations to date.

The authors thank the two anonymous reviewers for their helpful feedback and comments. 
Funding information Open access funding provided by Max Planck Society. This study is supported by the German Federal Ministry for Economic Affairs and Energy (BMWi), in the joint project: "MathEnergy - Mathematical Key Technologies for Evolving Energy Grids," sub-project: Model Order Reduction (Grant No. 0324019B).

Code availability section The source code of the presented numerical examples can be obtained from: http://runmycode.org/companion/view/3270 and is authored by: CHRISTIAN HIMPE.

\section{Compliance with ethical standards}

Conflict of interest The authors declare that they have no conflict of interest.

Open Access This article is distributed under the terms of the Creative Commons Attribution 4.0 International License (http://creativecommons.org/licenses/by/4.0/), which permits unrestricted use, distribution, and reproduction in any medium, provided you give appropriate credit to the original author(s) and the source, provide a link to the Creative Commons license, and indicate if changes were made.

\section{References}

1. Antoulas, A.C.: Approximation of Large-Scale Dynamical Systems, Adv. Des. Control, vol. 6. SIAM Publications, Philadelphia (2005). https://doi.org/10.1137/1.9780898718713

2. Baur, U., Benner, P., Feng, L.: Model order reduction for linear and nonlinear systems: a systemtheoretic perspective. Arch. Comput. Methods Eng. 21(4), 331-358 (2014). https://doi.org/10.1007/ s11831-014-9111-2

3. Benner, P.: Solving large-scale control problems. IEEE Control Syst Mag. 14(1), 44-59 (2004). https://doi.org/10.1109/MCS.2004.1272745

4. Benner, P., Himpe, C., Mitchell, T.: On reduced input-output dynamic mode decomposition. Adv. Comput. Math. 44(6), 1821-1844 (2018). https://doi.org/10.1007/s10444-018-9592-X

5. Benner, P., Kürschner, P.: Computing real low-rank solutions of Sylvester equations by the factored ADI method. Comput. Math. Appl. 67(9), 1656-1672 (2014). https://doi.org/10.1016/j.camwa.2014. 03.004

6. Benner, P., Kürschner, P., Saak, J.: Self-generating and efficient shift parameters in ADI methods for large Lyapunov and Sylvester equations. Electron. Trans. Numer. Anal. 43, 142-162 (2014). http:// etna.mcs.kent.edu/volumes/2011-2020/vol43/abstract.php?vol=43\&pages=142-162

7. Benner, P., Li, R.C., Truhar, N.: On the ADI method for Sylvester equations. J. Comput. Appl. Math. 233(4), 1035-1045 (2009). https://doi.org/10.1016/j.cam.2009.08.108

8. Bond, B.N., Daniel, L.: Guaranteed stable projection-based model reduction for indefinite and unstable linear systems. In: 2008 IEEE/ACM International Conference on Computer-Aided Design (2008). https://doi.org/10.1109/ICCAD.2008.4681657

9. Bru, R., Coll, C., Thome, N.: Symmetric singular linear control systems. Appl. Math. Lett. 15(6), 671-675 (2002). https://doi.org/10.1016/S0893-9659(02)00026-5

10. Chahlaoui, Y., Van Dooren, P.: A collection of benchmark examples for model reduction of linear time invariant dynamical systems. Tech. Rep. 2002-2, SLICOT Working Note. Available from http:// www.slicot.org (2002)

11. Davidson, A.: Balanced systems and model reduction. Electron. Lett. 22(10), 531-532 (1986). https://doi.org/10.1049/el:19860362

12. Fernando, K.V., Nicholson, H.: Minimality of SISO linear systems. Proc. IEEE 70(10), 1241-1242 (1982). https://doi.org/10.1109/PROC.1982.12460

13. Fernando, K.V., Nicholson, H.: On the structure of balanced and other principal representations of SISO systems. IEEE Trans. Autom. Control 28(2), 228-231 (1983). https://doi.org/10.1109/TAC. 1983.1103195

14. Gardiner, J.D., Laub, A.J., Amato, J.J., Moler, C.B.: Solution of the Sylvester matrix equation $A X B+$ $C X D=E$. ACM Trans. Math. Softw. 18(2), 223-231 (1992). https://doi.org/10.1145/146847.146 929 
15. Gugercin, S., Antoulas, A.C., Beattie, C.: $\mathcal{H}_{2}$ model reduction for large-scale linear dynamical systems. SIAM J. Matrix Anal. Appl. 30(2), 609-638 (2008). https://doi.org/10.1137/060666123

16. Himpe, C.: emgr - the Empirical Gramian Framework. Algorithms 11(7), 91 (2018). https://doi.org/ 10.3390/a11070091

17. Himpe, C.: emgr - EMpirical GRamian framework (version 5.7) (2019). https://gramian.de. https:// doi.org/10.5281/zenodo. 2577980

18. Himpe, C., Leibner, T., Rave, S.: Hierarchical approximate proper orthogonal decomposition. SIAM J. Sci. Comput. 40(5), A3267-A3292 (2018). https://doi.org/10.1137/16M1085413

19. Himpe, C., Leibner, T., Rave, S., Saak, J.: Fast low-rank empirical cross Gramians. Proc. Appl. Math. Mech. 17(1), 841-842 (2017). https://doi.org/10.1002/pamm.201710388

20. Himpe, C., Ohlberger, M.: A note on the cross Gramian for non-symmetric systems. Syst. Sci. Control Eng. 4(1), 199-208 (2016). https://doi.org/10.1080/21642583.2016.1215273

21. Himpe, C., Rave, S.: HAPOD - hierarchical approximate proper orthogonal decomposition (version 2.0). https://git.io/hapod (2019)

22. Jiang, Y.L., Qi, Z.Z., Yang, P.: Model order reduction of linear systems via the cross Gramian and SVD. IEEE Trans. Circ. Syst. II: Express Briefs 66(3), 422-426 (2019). https://doi.org/10.1109/ TCSII.2018.2864115

23. Li, J.R., White, J.: Efficient model reduction of interconnect via approximate system Gramians. In: 1999 IEEE/ACM International Conference on Computer-Aided Design. Digest of Technical Papers, pp. 380-383 (1999). https://doi.org/10.1109/ICCAD.1999.810679

24. Li, J.R., White, J.: Reduction of large circuit models via low rank approximate Gramians. Int. J. Appl. Math. Comput. Sci. 11(5), 1151-1171 (2001). http://eudml.org/doc/207549

25. The MathWorks, Inc., http://www.matlab.com: MATLAB

26. Mirsky, L.: A trace inequality of John von Neumann. Monat. Math. 79(4), 303-306 (1975). https://doi.org/10.1007/BF01647331

27. Moore, B.C.: Principal component analysis in linear systems: controllability, observability, and model reduction. IEEE Trans. Autom. Control AC-26(1), 17-32 (1981). https://doi.org/10.1109/TAC.1981. 1102568

28. Moosmann, C., Greiner, A.: Convective thermal flow problems. In: Dimension Reduction of LargeScale Systems, vol. 45, pp. 341-343. Springer (2005). https://doi.org/10.1007/3-540-27909-1_16

29. Opmeer, M.R., Reis, T.: A lower bound for the balanced truncation error for MIMO systems. IEEE Trans. Autom. Control 60(8), 2207-2212 (2015). https://doi.org/10.1109/TAC.2014.2368232

30. Or, A.C., Speyer, J.L., Kim, J.: Reduced balancing transformations for large nonnormal state-space systems. J. Guid. Control Dyn. 35(1), 129-137 (2012). https://doi.org/10.2514/1.53777

31. Peng, L., Mohseni, K.: Symplectic model reduction of Hamiltonian systems. SIAM J. Sci. Comput. 38(1), A1-A27 (2016). https://doi.org/10.1137/140978922

32. Penzl, T.: Algorithms for model reduction of large dynamical systems. Linear Algebra Appl. 415(2-3), 322-343 (2006). https://doi.org/10.1016/j.laa.2006.01.007. (Reprint of Technical Report SFB393/9940, TU Chemnitz, 1999.)

33. Perev, K.: The unifying feature of projection in model order reduction. Inf. Technol. Control 12(3-4), 17-27 (2016). https://doi.org/10.1515/itc-2016-0003

34. Rahrovani, S., Vakilzadeh, M.K., Abrahamsson, T.: On Gramian-based techniques for minimal realization of large-scale mechanical systems. In: Topics in Modal Analysis, vol. 7, pp. 797-805 (2014). https://doi.org/10.1007/978-1-4614-6585-0_75

35. Redmann, M., Kürschner, P.: An output error bound for time-limited balanced truncation. Syst. Control Lett. 121, 1-6 (2018). https://doi.org/10.1016/j.sysconle.2018.08.004

36. Rowley, C.W.: Model reduction for fluids, using balanced proper orthogonal decomposition. Int. J. Bifurcat. Chaos 15(3), 997-1013 (2005). https://doi.org/10.1142/S0218127405012429

37. Saak, J.: Efficient numerical solution of large scale algebraic matrix equations in PDE control and model order reduction. Dissertation, Technische Universität Chemnitz, Chemnitz. http:// nbn-resolving.de/urn:nbn:de:bsz:ch1-200901642 (2009)

38. Shaker, H.R.: Generalized cross-Gramian for linear systems. In: Proceedings of IEEE Conf. Ind. Electron. Appl., pp. 749-751 (2012). https://doi.org/10.1109/ICIEA.2012.6360824

39. Shi, G., Shi, C.R.J.: Model-order reduction by dominant subspace projection: error bound, subspace computation, and circuit applications. IEEE Trans. Circ. Syst. I: Reg. Papers 52(5), 975-993 (2005). https://doi.org/10.1109/TCSI.2005.846217 
40. Sorensen, D.C., Antoulas, A.C.: The Sylvester equation and approximate balanced reduction. Numer. Lin. Alg. Appl. 351-352, 671-700 (2002). https://doi.org/10.1016/S0024-3795(02)00283-5

41. Stykel, T.: Gramian-based model reduction for descriptor systems. Math. Control Signal. Syst. 16(4), 297-319 (2004). https://doi.org/10.1007/s00498-004-0141-4

42. The MORwiki Community: MORwiki - Model Order Reduction Wiki. http://modelreduction.org

43. Toscano, R.: Structured controllers for uncertain systems. Advances in industrial control. Springer, London (2013). https://doi.org/10.1007/978-1-4471-5188-3

44. Wang, X., Yu, M.: The error bound of timing domain in model order reduction by Krylov subspace methods. J. Circ. Syst. Comput. 27(6), 1850093 (2018). https://doi.org/10.1142/S0218126618500937

45. Willcox, K., Peraire, J.: Balanced model reduction via the proper orthogonal decomposition. AIAA J. 40(11), 2323-2330 (2002). https://doi.org/10.2514/2.1570

46. Wolf, T., Panzer, H., Lohmann, B.: Gramian-based error bound in model reduction by Krylov subspace methods. IFAC Proc. Vol. (Proc. 18th IFAC World Congress) 44(1), 3587-3592 (2011). https://doi.org/10.3182/20110828-6-IT-1002.02809

47. Wong, N.: Efficient positive-real balanced truncation of symmetric systems via cross-Riccati equations. IEEE Trans. Comput.-Aided Des. Integr. Circ. Syst. 27(3), 470-480 (2008). https://doi.org/10. 1109/TCAD.2008.915534

Publisher's note Springer Nature remains neutral with regard to jurisdictional claims in published maps and institutional affiliations. 